\title{
DEVELOPMENTAL STUDIES OF OLIGODENDROCYTE PRECURSOR CELL MIGRATION AND THEIR IMPLICATIONS FOR TRANSPLANTATION AS THERAPY FOR MULTIPLE SCLEROSIS
}

\author{
CHARLES FFRENCH-CONSTANT $^{1}$, B. W. KIERNAN ${ }^{2}$, RICHARD MILNER ${ }^{1}$ \\ and SUZANNA SCOTT-DREW ${ }^{1}$ \\ Cambridge
}

Multiple sclerosis (MS) is a common and devastating neurological disease. With a prevalence of approximately $1 / 1000$ it represents a major cause of disability in the United Kingdom. Ophthalmic symptoms are common, with optic neuritis leading to grossly impaired visual acuity and brain stem lesions producing ophthalmoplegia. These symptoms add to the problems caused by damage within motor systems, leading to the familiar clinical picture of a patient disabled by a combination of poor vision and motor dysfunction.

The pathogenesis of MS is poorly understood. A generally accepted view is that MS is an autoimmune disease in which T-cell stimulation, resulting from the presentation of unknown antigens by macrophages within the brain, initiates the destruction of oligodendrocytes and their myelin sheaths. ${ }^{1-3}$ As a consequence, rapid nerve conduction is lost and symptoms result. Until recently there was no effective therapy. However, insights into the mechanisms of T-cell stimulation have suggested therapies to suppress T-cell activity and so reduce demyelination. One form of treatment, interferon- $\beta$, looks particularly promising in early trials ${ }^{4}$ but caution is required at this stage.

Even with effective therapy to reduce T-cell stimulation in MS, many sufferers will be left with extensive demyelinated lesions in which there is little or no inflammatory activity but no repair. These lesions will therefore continue to produce symptoms even in individuals whose disease is quiescent. An important therapeutic goal will then be the repair of such damage once the disease is under control. The recent demonstration that transplanted oligodendroglial cells will remyelinate lesions in the rat spinal

From: Wellcome/CRC Institute of Developmental Biology and Cancer and Departments of 'Pathology, and ${ }^{2}$ Zoology, University of Cambridge, Cambridge, UK.

Correspondence to: Dr Charles ffrench-Constant, Wellcome/CRC Institute, Tennis Court Road, Cambridge CB2 1QR, UK. cord $^{5}$ suggests that transplantation may provide an effective method for such repair. The purpose of this review is to highlight some of the potential problems in transplantation-based strategies for MS and discuss how knowledge of the developmental biology of myelination suggests ways of overcoming these problems.

The most obvious problem when considering transplantation for MS is that MS is a multifocal disease. This point is well illustrated by the human optic nerve shown in Fig. 1. As can be seen, there are a number of patches of demyelination along the nerve, each separated by normal myelinated regions. This pattern will be repeated throughout the affected areas within the central nervous system (CNS), making it impossible to place transplanted cells into each individual lesion. Rather, groups of lesions will have to be targeted by each individual transplant. This in turn will require that the transplanted cells have the ability to migrate into the demyelinated areas prior to forming new myelin.

Cell culture studies of oligodendroglial development in rodents suggest that a defined oligodendrocyte precursor cell possesses these migratory properties and may therefore be most appropriate for transplantation. These studies have shown that, while the mature oligodendrocyte is a non-motile cell, the precursor is highly migratory. ${ }^{6}$ Moreover, two sets of experiments in vivo suggest that these cells can migrate extensively within the normal CNS.

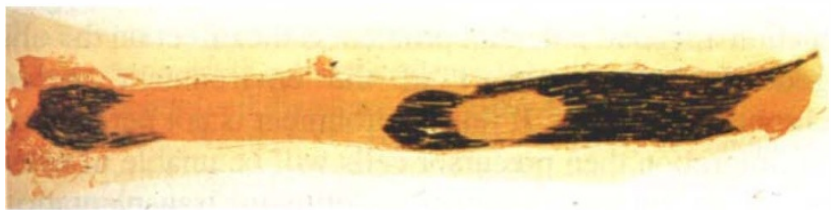

Fig. 1. A longitudinal section through a human optic nerve showing typical features of MS. Myelin is stained black; note the patches of demyelination with normal myelin in between. (Reproduced with permission from ffrench-Constant. ${ }^{2}$ ) 
First, Levison and Goldman ${ }^{7}$ injected replication-deficient retroviruses expressing the $\beta$-galactosidase gene into the subventricular zone (SVZ) of neonatal rats. The SVZ is thought to be the site of origin of oligodendrocyte precursors in the postnatal rat brain. Following injection, migration of labelled cells from the SVZ occurred into both white and grey matter of the ipsilateral hemisphere. Many of the labelled cells were oligodendrocytes, confirming directly the migration of cells of the oligodendroglial lineage. Second, Warrington et al. ${ }^{8}$ separated oligodendrocyte precursors from mature oligodendrocytes and then transplanted these different cell populations into shiverer mutant mice. In this system, the formation of myelin by transplanted cells can be analysed by immunostaining for myelin basic protein (MBP) which is lacking in the shiverer host as a result of a deletion within the MBP gene. Extensive migration was seen only in the animals transplanted with the immature oligodendrocyte precursors. Animals transplanted with the mature cells showed smaller areas of myelin close to the site of the transplant, consistent with a diminished capacity for migration of the mature oligodendrocytes.

Taken together, these results suggest that the oligodendrocyte precursor represents an ideal candidate cell for transplantation into the CNS of MS sufferers. However, the distances required for migration in patients will be much greater than those seen during development, as the embryonic CNS is obviously much smaller than that in the adult. For this reason, it may be necessary to enhance the migration of these cells prior to transplantation. Appropriate manipulation might involve overexpression or 'knocking out' of molecules that respectively stimulate or inhibit migration during normal development. In order to identify such molecules, we have begun an analysis of the cell-extracellular matrix (ECM) interactions that might mediate oligodendrocyte precursor migration, as these interactions play a critical role in cell migration elsewhere during development. ${ }^{9}$ Our preliminary results show that oligodendrocyte precursors express molecules of the integrin family that are known to act as ECM receptors, ${ }^{10}$ consistent with a role for cell-ECM interactions in oligodendroglial migration. The next step is to determine the integrins specifically associated with migration and then overexpress these so as to enhance the normal migratory potential of oligodendroglial cells. Such enhanced cells could then be tested in transplantation studies with animal models of MS.

Once transplanted cells have reached areas of demyelination a second potential problem is the effect on the oligodendrocyte precursors of the environment within chronic MS lesions. If this environment is not permissive for migration then precursor cells will be unable to enter the lesion and myelin formation following transplantation will be impaired. One particular concern is the astrocyte hyperplasia that characterises chronic MS lesions. ${ }^{11}$ Astrocytes have been implicated in the inhibition of axon outgrowth in the mature $\mathrm{CNS}^{12}$ and may have similar effects on migrating oligodendrocyte precursor cells. The dense network of astrocyte processes in MS lesions might therefore present a barrier to precursor migration and so prevent repair.

Studies on the pathways of oligodendrocyte precursor cell migration during normal development show evidence for such barriers to migration. Oligodendrocyte precursor cells migrate along the optic nerve from the chiasm. ${ }^{6.13}$ In the rat these cells do not enter the retina and myelinate the retinal ganglion cell axons in the nerve fibre layer - they appear to be prevented from doing so by a barrier at the lamina cribrosa, the region of the nerve behind the eye where the nerve pierces the sclera. ${ }^{14}$ The effect of this is shown in Fig. 2; there is a short unmyelinated stretch of the rat optic nerve just behind the eye in the region of the barrier. As retinal ganglion cell axons are myelinated in the optic nerve it seems unlikely that this restriction of myelination is mediated by molecules on the axon surface. This was shown directly in a transplantation experiment by Perry and Lund ${ }^{15}$ in which retinae were transplanted into the brain. In these retinae, myelination was observed around the retinal ganglion cell axons in the nerve fibre layer, showing that the axons themselves do not inhibit myelination.

On the basis of the observation that radially orientated astrocytes are present in the lamina cribrosa region of the rat optic nerve but not in the rabbit optic nerve in which migrating precursor cells do enter the retina and form myelin, ffrench-Constant $e t$ al. ${ }^{14}$ proposed that the astrocytes in the rat lamina cribrosa formed a barrier to oligodendrocyte precursor migration. The molecular basis of this barrier is unknown. One attractive candidate molecule is the ECM glycoprotein tenascin. Tenascin has been reported as being present at high concentrations in this region of the adult mouse nerve. ${ }^{16}$ In collaboration with $\mathrm{Dr}$ Andreas Faissner (Heidelberg) we have found that tenascin inhibits oligodendrocyte precursor migration in vitro and might therefore prevent migration through this

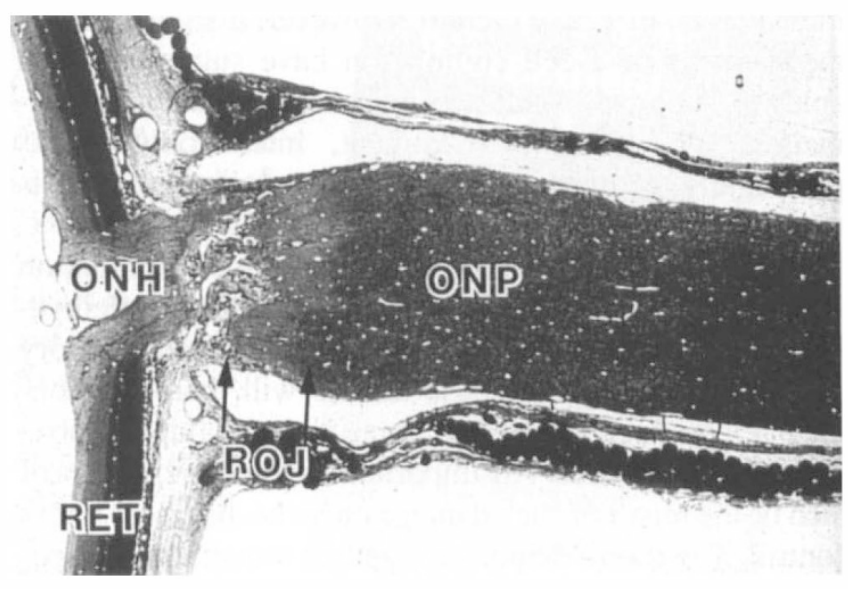

Fig. 2. A longitudinal section through a normal adult rat optic nerve, optic nerve head (ONH) and retina (RET). The darker shading of the nerve reflects the presence of myelin. Note that the optic nerve head is unmyelinated and pierced by blood vessels and other scleral components at the retina-optic nerve junction (ROJ). ONP, optic nerve proper.

(Reproduced with permission from Black et al..$^{22}$ ) 
region in vivo. The identification of putative extracellular barrier molecules is important for transplantation studies; such molecules are likely to be recognised by specific cell surface receptors and it follows that knocking out these receptors might make the transplanted cells unresponsive to inhibitory molecules. This in turn will lead to enhanced migration within previously inhibitory environments and, potentially, improved repair.

At this stage we do not know the extent to which the two problems discussed above - failure of migration within the unaffected CNS between lesions and failure to migrate into the lesion itself - will be significant factors in preventing repair following transplantation in MS. Answers to this question will come from the initial transplantation studies using unmodified cells. However, valuable clues may emerge from an examination of the distribution of oligodendrocyte precursors in MS patients. Oligodendrocyte precursors are present in the normal adult rat $\mathrm{CNS}^{17.18}$ and therefore are probably also present in the adult human CNS. Analysis of MS lesions has shown that there is a considerable degree of spontaneous repair by proliferating oligodendroglial cells. ${ }^{19,20}$ These cells are presumably oligodendrocyte precursors, as mature oligodendrocytes possess little if any proliferative capacity. ${ }^{21} \mathrm{~A}$ comparison of the distribution of these cells in MS patients and normal controls will define the extent of precursor cell migration towards and into MS lesions. These studies may show extensive redistribution of precursor cells into the lesions, suggesting that the migration of these cells is already adequate and that transplantation of unmodified cells will be appropriate. Alternatively, they may show no redistribution of precursor cells, suggesting that migration within the CNS between lesions is prevented; finally, they may show a pattern of redistribution surrounding but not within lesions, suggesting that migration towards lesions is adequate while entry into the lesion is inhibited. In either of the last two scenarios, manipulation of transplanted cells would be expected to enhance repair and a complete analysis of the developmental biology of oligodendrocyte precursors will allow potential candidate molecules to be identified.

The authors are grateful to Drs Bill Blakemore, Robin Franklin and Jack Price for helpful discussions, and to Dr Greta Mathews for comments on the manuscript. The work described is funded by the Wellcome Trust. C.ff.C. is a Wellcome Trust Senior Clinical Fellow, R.M. is a Wellcome Trust Prize Student and B.W.K. is an SERC Student.

\section{REFERENCES}

1. Bernard CCA, de Rosbo NK. Multiple sclerosis: an autoimmune disease of multifactorial aetiology. Curr Opin Immunol 1992;4:760-5.

2. ffrench-Constant C. Pathogenesis of multiple sclerosis. Lancet 1994;343:271-5.

3. Raine CS. Multiple sclerosis: a pivotal role for the $\mathrm{T}$ cell in lesion development. Neuropathol Appl Neurobiol 1991;17: 265-74.

4. IFNB Multiple Sclerosis Study Group. Interferon beta-1b is effective in relapsing-remitting multiple sclerosis. I. Clinical results of a multicentre, randomised, double-blind, placebo-controlled trial. Neurology 1993;43:655-61.

5. Groves AK, Barnett SC, Franklin RJM, Crang AJ, Mayer M, Blakemore WF, Noble M. Repair of demyelinated lesions by transplantation of purified O-2A progenitor cells. Nature 1993;362:453-5.

6. Small RK, Riddle P, Noble M. Evidence for migration of oligodendrocyte-type- 2 astrocyte progenitor cells into the developing rat optic nerve. Nature 1987;328:155-7.

7. Levison SW, Goldman JE. Both oligodendrocytes and astrocytes develop from progenitors in the subventricular zone of postnatal rat forebrain. Neuron 1993;10:201-12.

8. Warrington AE, Barbarese E, Pfeiffer SE. Differential myelinogenic capacity of specific developmental stages of the oligodendrocyte lineage upon transplantation into hypomyelinating hosts. J Neurosci Res 1993;34:1-13.

9. Adams JC, Watt FM. Regulation of development and differentiation by the extracellular matrix. Development 1993; 117:1183-98.

10. Hynes RO. Integrins: versatility, modulation and signalling in cell adhesion. Cell 1992;69:11-25.

11. Allen IV. Pathology of multiple sclerosis. In: Matthews WB, editor. McAlpine's multiple sclerosis. Edinburgh: Churchill Livingstone, 1991:341-78.

12. Liuzzi FJ, Lasek RJ. Astrocytes block axonal regeneration in mammals by activating the physiological stop pathway. Science 1987;237:642-5.

13. Mudhar HS, Pollock RA, Wang C, Stiles CD, Richardson WD. PDGF and its receptors in the developing rodent retina and optic nerve. Development 1993;118:539-52.

14. ffrench-Constant C, Miller RH, Burne JF, Raff MC. Evidence that migratory oligodendrocyte-type- 2 astrocyte $(\mathrm{O}-2 \mathrm{~A})$ progenitor cells are kept out of the rat retina by a barrier at the eye-end of the optic nerve. J Neurocytol 1988;17: 13-25.

15. Perry VH, Lund RD. Evidence that the lamina cribrosa prevents intraretinal myelination of retinal ganglion cell axons. J Neurocytol 1990;19:265-7 2.

16. Bartsch U, Bartsch S, Dörries U, Schachner M. Immunohistological localisation of tenascin in the developing and lesioned adult mouse optic nerve. Eur J Neurosci 1992;4: 338-52.

17. ffrench-Constant C, Raff MC. Proliferating bipotential glial progenitor cells in adult rat optic nerve. Nature 1986;319: 499-502.

18. Wolswijk G, Noble M. Identification of an adult-specific glial progenitor cell. Development 1989;105:387-400.

19. Prineas JW, Barnard RO, Kwon EE, Sharer LR, Cho E-S. Multiple sclerosis: remyelination of nascent lesions. Ann Neurol 1993;33:137-51.

20. Prineas JW, Kwon EE, Goldenberg PZ, Ilyas AA, Quarles RH, Benjamins JA, Sprinkle TJ. Multiple sclerosis: oligodendrocyte proliferation and differentiation in fresh lesions. Lab Invest 1989;61:489-503.

21. Temple S, Raff MC. Clonal analysis of oligodendrocyte development in culture: evidence for a developmental clock that counts cell divisions. Cell 1986;44:773-9.

22. Black JA, Waxman SG, Hildebrand C. Axo-glial relations in the retina-optic nerve junction of the adult rat: freeze-fracture observi, ions on axon membrane. J Neurocytol 1985; 14 : 887-907. 